\title{
Polypoid gallbladder neuroendocrine tumor diagnosed as benign polyp before surgery: A case report
}

\author{
SHIGETOSHI NAITO ${ }^{1,2}$, MASAYASU NAITO ${ }^{2}$, NOBUHARU YAMAMOTO ${ }^{2}$, \\ TOHRU KUME ${ }^{2}$, SEIICHIROU HOSINO ${ }^{2}$, YOSHINAO KINJYO ${ }^{3}$, YOSHIKI NAITO ${ }^{3}$, \\ HISANORI NAITO $^{2}$ and SUGURU HASEGAWA ${ }^{1}$ \\ ${ }^{1}$ Department of Gastroenterological Surgery, Faculty of Medicine, Fukuoka University, Jonan-ku, Fukuoka 814-0180; \\ ${ }^{2}$ Naito Hospital, Kurume, Fukuoka 830-0038; ${ }^{3}$ Department of Pathology, Kurume University School of Medicine, \\ Kurume, Fukuoka 830-0011, Japan
}

Received April 5, 2019; Accepted November 25, 2019

DOI: $10.3892 / \mathrm{mco} .2019 .1971$

\begin{abstract}
Gallbladder neuroendocrine tumors (GB-NETs) comprise only $0.5 \%$ of all NET cases, and their biology has been incompletely characterized. In the present study we report the case of a 50-year-old male patient with GB-NET who was admitted to Naito Hospital with diarrhea as the main complaint. At initial diagnosis, serum carcinoembryonic antigen (CEA) and carbohydrate antigen 19-9 (CA19-9) levels were within the normal range. Abdominal ultrasonography and contrast-enhanced computed tomography (CT) revealed gallbladder adenomyomatosis and cholecystitis, and an 8-mm pedunculated polypoid lesion was found in the neck of the gallbladder using drip infusion cholecystocholangiography-CT. As it was considered a benign polyp, laparoscopic cholecystectomy was performed. Pathological examination revealed a polypoid lesion that comprised NET cells with a cord-like or ribbon-like arrangement, and the cells exhibited positive immunostaining for chromogranin A and synaptophysin. In addition, immunohistochemical staining showed a Ki-67 index (i.e., proliferation index) of $<1 \%$, and no necrosis or mitotic figures were observed in the background. Based on these observations, we diagnosed the following: GB-NET, $\mathrm{G} 1,10 \times 12 \mathrm{~mm}$ in size and located in the gallbladder neck. According to the World Health Organization 2010 classification, NET G1 is a well-differentiated tumor, with the tumor
\end{abstract}

Correspondence to: Dr Shigetoshi Naito, Department of Gastroenterological Surgery, Faculty of Medicine, Fukuoka University, 7-45-1 Nanakuma, Jonan-ku, Fukuoka 814-0180, Japan

E-mail: shinaito@fukuoka-u.ac.jp

Abbreviations: CEA, carcinoembryonic antigen; CA19-9, carbohydrate antigen 19-9; CT, computed tomography; GB-NET, gallbladder neuroendocrine tumors; NEC, neuroendocrine carcinoma; US, ultrasound examination; RAS, Rokitansky-Ashoff sinus; DIC-CT, drip infusion cholecystocholangiography-computed tomography

Key words: gallbladder, laparoscopic cholecystectomy, polypoidneuroendocrine tumor, Ki-67, drip infusion cholecystocholangio-graphy-CT cells having a low proliferative potential $[\mathrm{Ki}-67$ index $\leq 2 \%$; mitotic figure number $<2$ (/10 HPF)]. It is regarded as a low- to mild-grade malignancy. Low-grade GB-NET occurs relatively rarely, and no clear guidelines have been formulated regarding its surgical treatment, such as minimal surgical excision margins or lymph node dissection. Detailed treatment recommendations should be developed after systematic studies of additional cases of GB-NET.

\section{Introduction}

Neuroendocrine cell tumor is considered a carcinoid and recognized as a benign tumor (1). However, as a result of subsequent research, it has come to light that neuroendocrine tumors (NETs) of the digestive system are malignant (2). Gallbladder NETs (GB-NETs) are rare, accounting for only $0.5 \%$ of all tumors (3). It is usually difficult to diagnose a GB-NET based on ultrasound (US) examination, abdominal computed tomography (CT), and abdominal magnetic resonance imaging (MRI) (4). GB-NETs are often detected during pathological examination after cholecystectomy performed for other conditions, such as cholelithiasis or gallbladder benign polyps $(5,6)$. In addition, it is difficult to diagnose GB-NET by only conducting preoperative examination.

The current WHO classification of NETs divides them into subgroups: Low-grade tumors are classified as NET G1, which exhibit benign behavior; medium-grade tumors as NET G2; high-grade tumors as neuroendocrine carcinoma (NEC); and tumors comprising normal adenocarcinoma and NET components as mixed adenoendocrine carcinoma (7). A majority of GB-NETs are poorly differentiated and exhibit increased mitotic activity and clinically aggressive course (3). However, NET G1, which has a benign behavior, is extremely rare in the gallbladder. Herein, we report the case of a patient with polypoid GB-NET G1 of the gallbladder and discuss the development of therapy for this tumor.

\section{Case report}

A 50-year-old male was admitted to Naito Hospital with diarrhea as the main complaint. No abnormalities were 

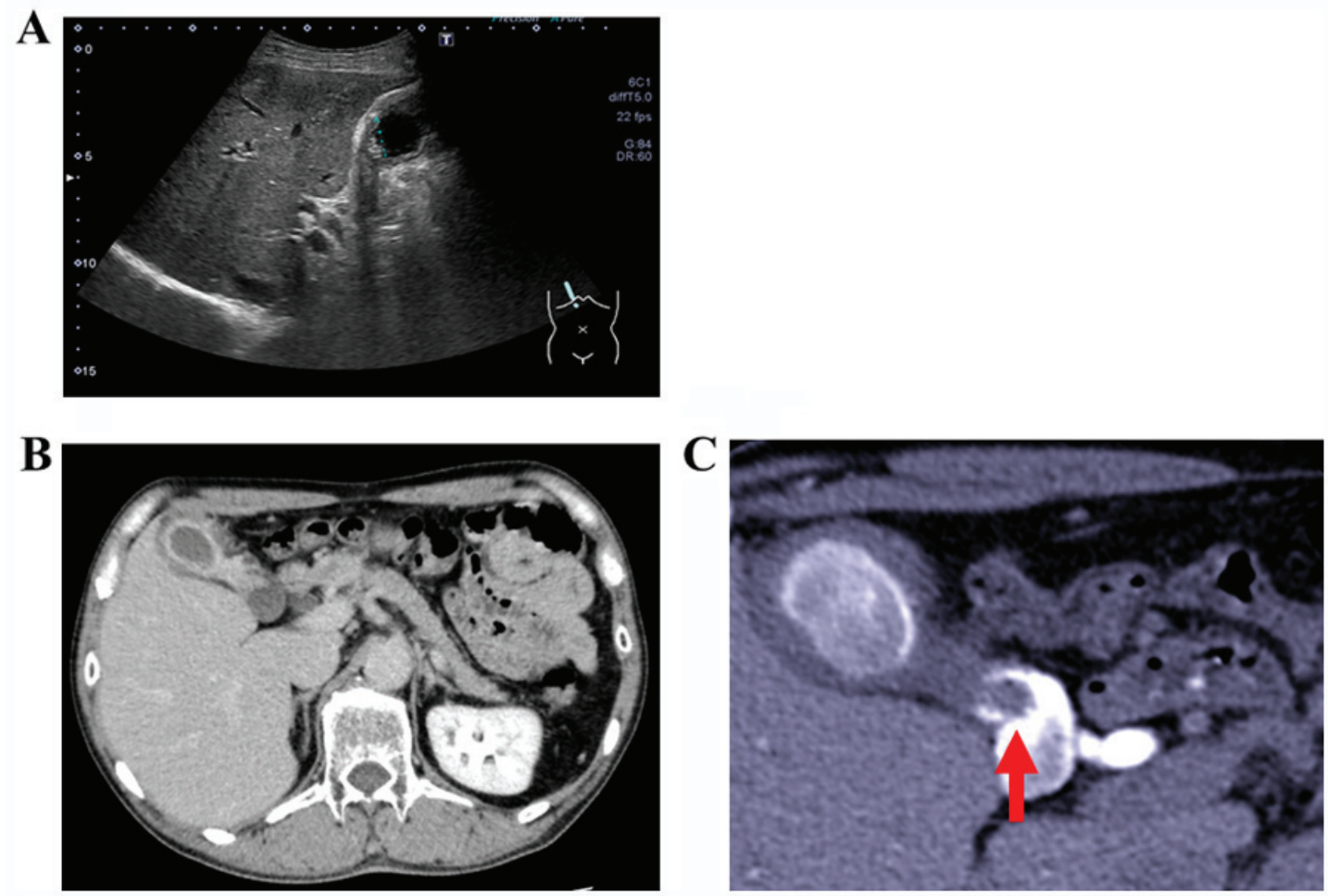

Figure 1. (A) US image revealing gall bladder wall thickening with edematous changes and the presence of multiple gallbladder stones. (B) A contrast-enhanced computed tomography image indicating wall thickening, similar to the findings obtained on US. (C) Drip infusion cholecystocholangiography-computed tomography image revealing the shadow defect (arrow) in the neck area of the gallbladder, with suspected presence of gallbladder stones or benign polyp. US, ultrasonography.

found, particularly in the thoracoabdominal region. Laboratory examination revealed elevated white blood cell counts $(11,760 / \mu 1$; normal range: $3,500-9,000 / \mu 1)$. CEA and CA19-9 levels were within the normal range $(<37$ and $<5.0 \mathrm{U} / \mathrm{ml}$, respectively). Abdominal US examination revealed edematous wall thickening in the body and fundus of the gallbladder (Fig. 1A), raising suspicion of a cystic lesion. Rokitansky-Ashoff sinus (RAS) was observed in the thickened area. Some small stones were detected in the lumen of the RAS; the neck of gallbladder could not be clearly observed because of extensive wall thickening. A contrast-enhanced CT scan as well as abdominal US confirmed the presence of RAS and stones (Fig. 1B). Drip infusion cholecystocholangiography-CT (DIC-CT) revealed a pedunculated $8-\mathrm{mm}$ polyp in the gallbladder neck, with multiple stones visible in the gallbladder (Fig. 1C).

As the patient had not undergone routine abdominal examinations, we could not determine whether the polyp was growing. Generally, pedunculated polyps $<1 \mathrm{~cm}$ are considered to have low possibility of being cancerous. Thus, the patient was diagnosed as having a benign small gallbladder polyp and adenomyomatosis, and laparoscopic cholecystectomy was performed.

\section{Pathological findings}

Macroscopically, the gallbladder wall was thickened, and RAS could be observed clustered in the wall. These observations supported the diagnosis of diffuse gallbladder adenomyomatosis. In addition, a pedunculated

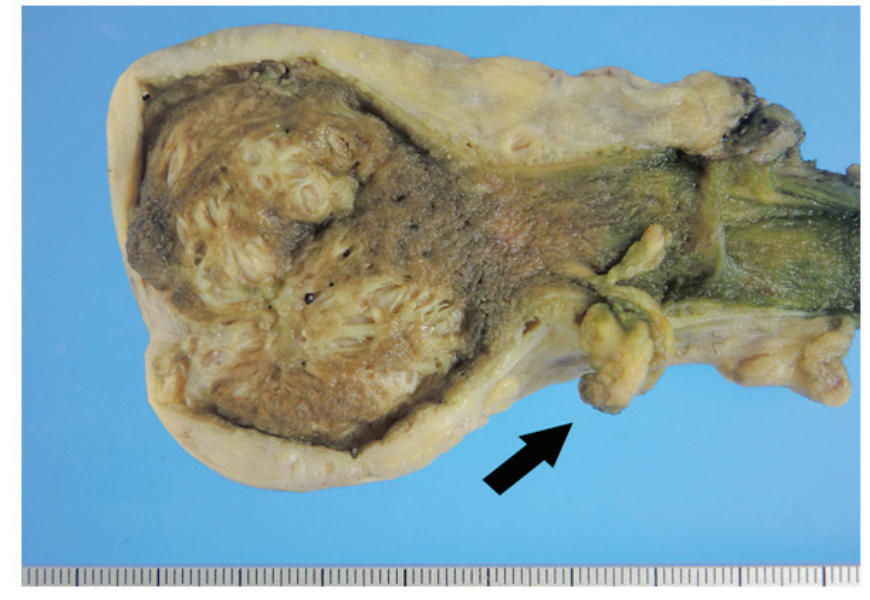

Figure 2. Surgically resected specimen: a polypoid tumor $(1.0 \times 1.2 \mathrm{~cm})$ located in the neck of the gallbladder (arrow).

polyp (10x12 mm) was observed in the neck of the gallbladder (Fig. 2).

Histopathological examination revealed that the polypoid lesion contained NET cells in a cord- or ribbon-like arrangement (Fig. 3A). The tumor cell morphology revealed isolated as well as disseminated small oval cells with high nucleus-to-cytoplasm ratios exhibiting a fine granular chromatin pattern (salt-and-pepper appearance) with slight aggregations, confirming the presence of small nucleoli (Fig. 3B). No necrosis or mitotic figures were observed in the background. Immunohistochemical staining with synaptophysin (Fig. 3C) and chromogranin A were 
A
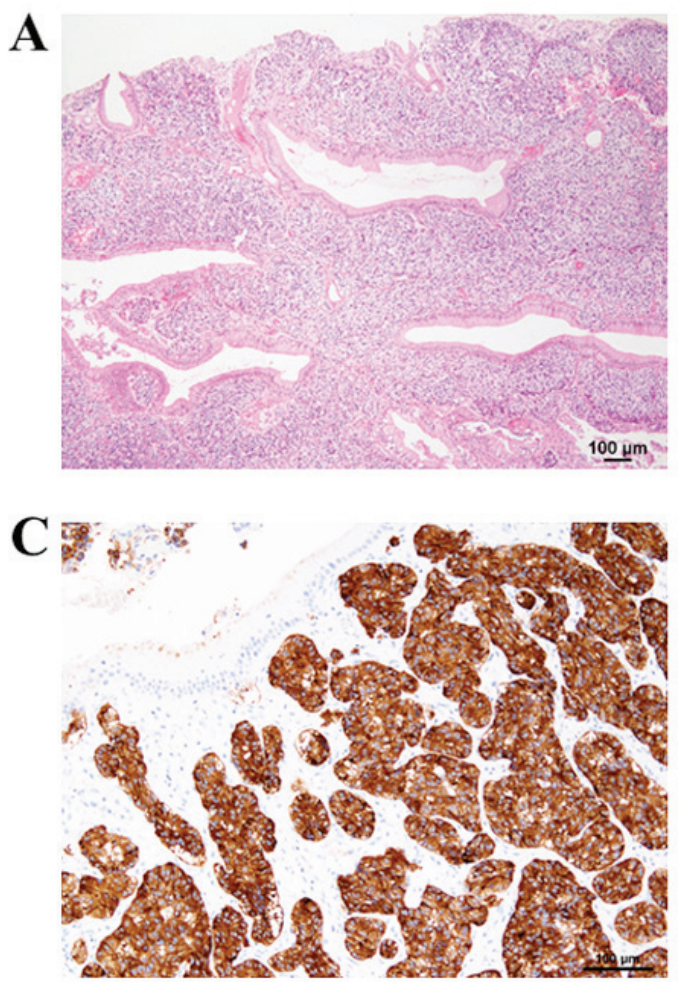

B

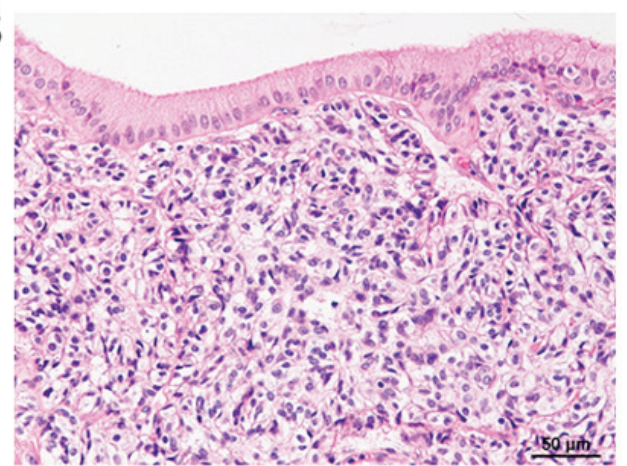

D

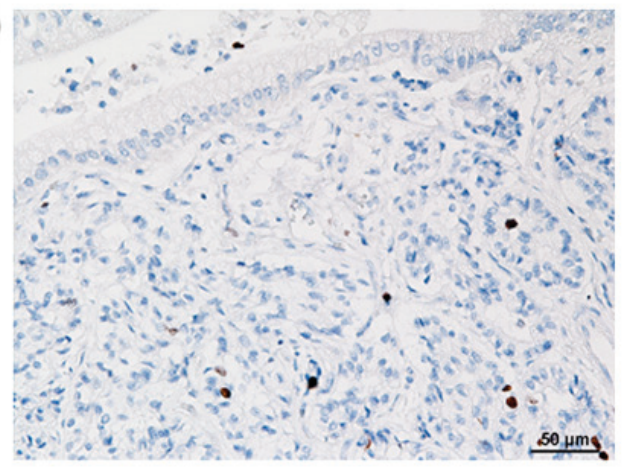

Figure 3. Hematoxylin and eosin staining. (A) Sub-epithelial and sub-mucosal proliferation of tumor cells in cord- or ribbon-like arrays. (B) Tumor cells had small round to oval nuclei with inconspicuous nucleoli. (C) Immunohistochemical staining of tumor cells with synaptophysin showing positive staining. (D) Immunohistochemical staining of tumor cells with Ki-67 showing a Ki-67 index $<1.0 \%$.

confined to the tumor, and Ki-67 labeling showed a labeling index of $<1 \%$ in the hot spot (Fig. 3D). Based on these findings, the patient was diagnosed as having polypoid GB-NET in the gallbladder neck area along with adenomyomatosis and cholecystitis. Tumor infiltration was limited to the submucosal layer, and there was no vascular invasion. We determined that because $\mathrm{R} 0$ resection was performed by cholecystectomy, no additional treatment was administered. The patient is alive and well without recurrence 1 year after the surgery.

\section{Discussion}

NETs originate from endocrine cells, and approximately $60 \%$ of the tumors occur in the gastrointestinal tract, with the frequency of occurrence being highest in the pancreas and rectum. Primary GB-NETs are rare, representing only $0.5 \%$ of all NETs (3). Therefore, little is known about their biological behavior (3). Neuroendocrine cells do not exist within the normal gall bladder or are present in small amounts in the epithelium. Various mechanisms have been proposed to explain the pathogenesis of GB-NET, and coexistence of cholelithiasis and gallbladder stones could be one such important factor (3). The proportion of neuroendocrine cells increases during chronic inflammation in the affected metaplastic epithelium, which may become the site of development of NETs (6). Generally, individuals with GB-NET do not exhibit any specific symptoms $(8,9)$, and the proportion of hormone-producing GB-NETs is unknown because of the small number of reported cases. According to previous findings from Japan, the percentage of pedunculated polyps in gall bladder NETs is higher than that in gastrointestinal NETs (10). However, it is difficult to distinguish GB-NETs from benign polyps because of the absence of specific findings on CT or MRI (4). Therefore, GB-NET is often misdiagnosed as benign prior to initial surgery $(5,6)$. In the present case, diagnosis was complicated owing to the presence of another gallbladder disease. Polypoid lesion was detected in the neck of the gallbladder on DIC-CT; however, the polyp was small $(8 \mathrm{~mm})$, and there were no other specific findings. Therefore, we could not establish the presence of a GB-NET using other physical and imaging techniques, including contrast-enhanced CT. In case of gastrointestinal NETs, other than those of the small bowel and pancreas, small tumors $(<2 \mathrm{~cm})$ and G1 tumors are believed to be successfully treated with local excision, even endoscopically, without lymph node dissection (11). The extent of surgery usually depends on the presence (or possibility) of lymph node metastasis. However, as the number of reported GB-NET cases is relatively small, risk factors for lymph node metastasis in GB-NET remain to be clarified. Yokoyama et al reported seven cases, two (28.6\%) of which had carcinoid tumors measuring $<1 \mathrm{~cm}$, with metastasis at presentation, whereas the remaining five cases had tumors measuring $\geq 3 \mathrm{~cm}$, with all five cases (100\%) developing metastases (12). These observations indicate that the risk of metastasis increases with increase in tumor size. Conversely, Hirose et al reported that even G1 tumors of extremely small size $(\leq 1 \mathrm{~cm})$ metastasize to the gallbladder lymph nodes, and extended additional surgery is required in such cases (13). In general, in cases of gastrointestinal and pancreatic NETs, high mitotic counts, high Ki-67 proliferation index 
values, large tumor diameters, and high invasion depths are considered risk factors for the existence of lymph node or distant metastasis (14-16). However, little is known about the risk factors for lymph node or distant metastasis in GB-NET cases. Furthermore, there is no evidence regarding the efficacy of extended surgery or adjuvant chemotherapy in GB-NET G1 and G2 cases (17). If metastasis is observed during the histopathological examination of the cystic duct node, it should be determined whether a radical second resection with regional lymphadenectomy is the appropriate treatment of choice (13). In cases with cystic duct lymph node metastasis, radical second resection with lymph node dissection should be considered as an option for accurate nodal evaluation, based on the reported outcomes of incidental gallbladder cancer (18). Our patient had NET-G1 with limited infiltration depth without lymph vascular invasion and lymph node metastasis. Therefore, he was closely followed up without adjuvant therapy. Regarding the prognosis, Eltawil et al reported that 5-year survival rates for tumors classified as carcinoids-neuroendocrine or small-cell carcinoma, are 36.9 and $0 \%$, respectively (5). By contrast, Koizumi et al reported that a tumor did not recur in $68.8 \%$ cases in which GB-NET was resected and that the average disease-free period of surviving cases was 28.7 months (range: 6-180 months). The substantial difference between the two aforementioned reports may be attributed to the difference in the pathology or stage of the tumor. The report by Eltawi et al could have included some case series with no stratification of the type of gallbladder NET and NEC. Some researchers advocate that in case of GB-NET, facilitated invasion and lymphatic metastasis to adjacent organs contribute to the high grade of malignancy (10). Further research is necessary to evaluate imaging findings prior to surgery, to determine the appropriate surgical procedure, and to identify the potential prognostic factors.

\section{Acknowledgements}

Not applicable.

\section{Funding}

No funding was received.

\section{Availability of data and materials}

All data generated or analyzed during the present study are included in this article.

\section{Authors' contributions}

SN collected the patient data and was a major contributor to writing the manuscript. MN contributed to analysis and interpretation of data, and assisted in the preparation of the manuscript. YN and YK performed the histological examination. SHo, NY, and TK were in charge of the operation of the patient in the hospital. HN, and SHa reviewed and contributed to the discussion and critical review of draft versions of the manuscript. All authors read and approved the final manuscript.

\section{Ethics approval and consent to participate}

Not applicable.

\section{Patient consent for publication}

The patient provided informed consent for publication of the data.

\section{Competing interests}

The authors declare that they have no competing interests.

\section{References}

1. La Rosa S, Bongiovanni M and Uccella S: Pathology of neuroendocrineneoplasms:Morphological,immunophenotypical, and circulating molecular markers. In: Atlas of thyroid and neuroendocrine tumor markers. Giovanella L. (eds). Springer, Cham, pp13-38, 2018.

2. Klöppel G, Perren A and Heitz PU: The gastroenteropancreatic neuroendocrine cell system and its tumors: The WHO classification. Ann N Y Acad Sci 1014: 13-27, 2004.

3. Eltawil KM, Gustafsson BI, Kidd M and Modlin IM: Neuroendocrine tumors of the gallbladder: An evaluation and reassessment of management strategy. J Clin Gastroenterol 44: 687-695, 2010.

4. Zou YP, Li WM, Liu HR and Li N: Primary carcinoid tumor of the gallbladder: A case report and brief review of the literature. World J Surg Oncol 8: 12, 2010.

5. Mezi S, Petrozza V, Schillaci O, La Torre V, Cimadon B, Leopizzi M, Orsi E and La Torre F: Neuroendocrine tumors of the gallbladder: A case report and review of the literature. J Med Case Rep 5: 334, 2011

6. Jun SR, Lee JM, Han JK and Choi BI: High-grade neuroendocrine carcinomas of the gallbladder and bile duct: Report of four cases with pathological correlation. J Comput Assist Tomogr 30: 604-609, 2006

7. Komminoth P, Arnold R, Capella C, Klimstra DS and Kloppel G. Neuroendocrine neoplasms of the gallbladder and extrahepatic bile ducts. In: Bosman FT, Carneiro F, Hruban RH, Theise HD, editors. WHO classification of tumours of the digestive system. Lyon: International Agency for Research on Cancer, pp. 274-276, 2010.

8. Monier A, Saloum N, Szmigielski W, Alrashid A and Napaki SM: Neuroendocrine tumor of the gallbladder. Pol J Radiol 80: 228-231, 2015.

9. Porter JM, Kalloo AN, Abernathy EC and Yeo CJ: Carcinoid tumor of the gallbladder: Laparoscopic resection and review of the literature. Surgery 112: 100-105, 1992.

10. Yotsumoto H, Godai T, Suematsu H, Yamauchi M, Fujikawa H, Fukano F, Tamura I, Rino Y, Suzuki S and Masuda M: A neuroendocrine tumor of the gallbladder. Jpn J Gastroenterol Surg 51: 263-270, 2018.

11. Kulke MH, Benson AB III, Bergsland E, Berlin JD, Blaszkowsky LS, Choti MA, Clark OH, Doherty GM, Eason J, Emerson L, et al: Neuroendocrine tumors. J Natl Compr Canc Netw 1: 724-764, 2012.

12. Yokoyama Y, Fujioka S, Kato K, Tomono H, Yoshida K and Nimura Y: Primary carcinoid tumor of the gallbladder: Resection of a case metastasizing to the liver and analysis of outcomes. Hepatogastroenterology 47: 135-139, 2000.

13. Hirose Y, Sakata J, Endo K, Takahashi M, Saito R, Imano H, Kido T, Yoshino K, Sasaki T and Wakai T: A 0.8-cm clear cell neuroendocrine tumor G1 of the gallbladder with lymph node metastasis: A case report. World J Surg Oncol 16: 150, 2018.

14. Saund MS, Al Natour RH, Sharma AM, Huang Q, Boosalis VA and Gold JS: Tumor size and depth predict rate of lymph node metastasis and utilization of lymph node sampling in surgically managed gastric carcinoids. Ann Surg Oncol 18: 2826-2832, 2011.

15. Sato Y, Hashimoto S, Mizuno K, Takeuchi M and Terai S: Management of gastric and duodenal neuroendocrine tumors. World J Gastroenterol 22: 6817-6828, 2016. 
16. Chatzipantelis P, Konstantinou P, Kaklamanos M, Apostolou G and Salla C: The role of cytomorphology and proliferative activity in predicting biologic behavior of pancreatic neuroendocrine tumors: A study by endoscopic ultrasound-guided fine-needle aspiration cytology. Cancer 17: 211-216, 2009.

17. National Comprehensive Cancer Network: NCCN Clinical Practice Guidelines in Oncology. Neuroendocrine tumors. version 1, 2011. http://www.lecba-rakoviny.cz/dokumenty/ NCCN_Guidelines_neuroendocrine_2011.pdf. Accessed December 18, 2019.
18. Wakai T, Shirai Y and Hatakeyama K: Radical second resection provides survival benefit for patients with T2 gallbladder carcinoma first discovered after laparoscopic cholecystectomy. World J Surg 26: 867-871, 2002.

c) (i) $\Theta$ This work is licensed under a Creative Commons EY NO NO Attribution-NonCommercial-NoDerivatives 4.0 International (CC BY-NC-ND 4.0) License. 